\title{
Sensitivity Analysis and Numerical Simulation of a SEIV Basic Dog-Rabies Mathematical Model with Control
}

\author{
Edwiga Renald $^{1}$, Dmitry Kuznetsov ${ }^{2}$, Katharina Kreppel ${ }^{3}$ \\ ${ }^{1,2}$ Department of Applied Mathematics and Computational Sciences, The Nelson Mandela African Institution of Science and \\ Technology, P. O. Box 447, Arusha Tanzania \\ ${ }^{3}$ Department of Global Health and Biomedical Sciences, The Nelson Mandela African Institution of Science and Technology, P. \\ O. Box 447, Arusha Tanzania
}

\begin{abstract}
Rabies is a zoonotic, viral disease that causes acute inflammation of the brain in humans and other mammals. It is transmitted by the saliva of infected animals via bites, scratches or contact between open body parts of infectious dog and noninfectious. In this paper, we have analysed a SEIV (Susceptible-Exposed-Infectious-Vaccinated) mathematical model for dog-rabies whereby sensitivity analysis and numerical simulation of the model have been carried out, presented and discussed. According to the sensitivity indices of parameters used at DFE (Disease Free Equilibrium) point, an infection rate of stray dogs is the most positive sensitive parameter while the natural death rate of stray dogs is the most negative sensitive parameter. These results lead us to the advice of putting much effort on these parameters when fighting against rabies disease transmission. Based on the numerical analysis results, it is advised to use mass vaccination of stray dogs as a strategy to fight against dog rabies transmission. Sensitivity analysis was done by using MATHEMATICA and the numerical simulation of the basic model was done by using ode45 MATLAB's standard solver for ordinary differential equations (ODEs). All analysis results were obtained by using data collected from Mbwa wa Africa and the Ministry of Agriculture, Livestock Development and Fisheries of the United Republic of Tanzania (URT).
\end{abstract}

Key Words: Effective Reproduction Number, Mathematical Modeling, Numerical Simulation, Rabies, Sensitivity Index

\section{INTRODUCTION}

Rabies is an acute progressive, fatal encephalomyelitis, transmitted most commonly through the bite of a rabid animal [1]. Globally, it consumes the life of approximately 61,000 human deaths worldwide [1]. Dogs are the most important rabies reservoir [2]. Rabies still is a worldwide one of the important health problems since it has became a re-emergent infection especially for the developing countries [3].

Mathematical modeling has historically been of great importance in epidemiology and is a useful tool for providing a better insight into the dynamics of epidemic diseases such as rabies.

In this paper, sensitivity analysis of the effective reproduction number based on parameters that have been used at the Disease Free Equilibrium (DFE) point has been carried out and numerical simulations of the basic SEIV (Susceptible-Exposed-InfectiousVaccinated) Mathematical model for dog-rabies have been presented and discussed.

In Mathematical modeling of Biological systems, sensitivity analysis plays an important role as it helps to determine how important each parameter is to disease transmission and also it helps to assess how sensitive a model is in relation to variations in the values of the model parameters and to changes in the general structure of the model [4]. It also helps to know which parameters are important in contributing to the prediction impression [4].

However, various Mathematical models have been proposed. Amongst others, these include models formulated by Hampson et al., [5], Lembo et al., [6], Zhang et al., [7], Hou et al., [8], Keller et al., [9], Ega et al., [10], Abta et al., [11], Elmore et al., [12], Ruan [13], Laager et al., [14], Chidumayo [15]. In this paper, an SEIV dog-rabies Mathematical model has been formulated. The model is based on urban areas near wild life reservoirs using Arusha region in Tanzania as an example. The model incorporates 
three dogs' sub-populations which are domestic dogs, stray dogs and Maasai dogs. Domestic dogs (canis familiaris) being defined as the dogs that live in a close relationship with human being [16], stray dogs being defined as the publicly roaming dogs [17] and Maasai dogs being defined as Livestock Guardian Dogs (LGDs) for Maasai tribe in Arusha region [18].

According to the sensitivity indices, infection rate of stray dogs $\beta_{s}$ is the most positive sensitive parameter followed by the annual births of stray $\operatorname{dogs} \alpha_{s}$ and the incubation period of stray dogs $\rho_{s}$. Conversely, natural death rate of stray dogs $\mu_{s}$ is the most negative sensitive parameter followed by death rate for stray dogs due to rabies $\delta_{\mathrm{s}}$ and vaccination rate of the susceptible stray $\operatorname{dogs} \sigma_{\mathrm{s}}$. From these results, it is advised that, putting much emphasis on the most positive and most negative sensitive parameters will be the most effective way in combating dog-rabies transmission in Arusha region.

Also, from the simulation results it is observed that, Applying dog mass vaccination will help to control the transmission. Culling will help at the moment it is practiced but after six months all culled dogs will get replaced by unvaccinated new born puppies.

Sensitivity analysis was done by using MATHEMATICA program while numerical simulation analysis results of the basic model were obtained through ode45 MATLAB's standard solver for Ordinary Differential Equations (ODEs).

\section{MATERIALS AND METHODS}

In this section, a formulated Mathematical model had been presented as a system of twelve (12) differential equations. The model consists of four (4) classes with every class being categorized into three (3) dogs' sub-population based on Arusha region. The four (4) classes are Susceptible, Exposed, Infectious and Vaccinated dogs while the three (3) sub-populations of dogs are domestic dogs, stray dogs and Maasai dogs. Below is the Model system.

$$
\left\{\begin{array}{c}
\frac{d S_{d}}{d t}=\alpha_{d}+\omega_{d} V_{d}+\Psi_{s d}+\Psi_{m d}-\mu_{d} S_{d}-\sigma_{d} S_{d}-\Psi_{d s}-\beta_{d} S_{d} I_{s} \\
\frac{d E_{d}}{d t}=\beta_{d} S_{d} I_{s}-\mu_{d} E_{d}-\rho_{d} E_{d} \\
\frac{d I_{d}}{d t}=\rho_{d} E_{d}-\left(\mu_{d}+\delta_{d}\right) I_{d} \\
\frac{d V_{d}}{d t}=\sigma_{d} S_{d}-\omega_{d} V_{d}-\mu_{d} V_{d} \\
\frac{d S_{s}}{d t}=\alpha_{s}+\omega_{s} V_{s}+\Psi_{d s}+\Psi_{m s}-\sigma_{s} S_{s}-\left(\mu_{s}+\mu_{c}\right) S_{s}-\Psi_{s d}-\beta_{s} S_{s} I_{s} \\
\frac{d E_{s}}{d t}=\beta_{s} S_{s} I_{s}-\mu_{s} E_{s}-\rho_{s} E_{s} \\
\frac{d I_{s}}{d t}=\rho_{s} E_{s}-\left(\mu_{s}+\delta_{s}\right) I_{s} \\
\frac{d V_{s}}{d t}=\sigma_{s} S_{s}-\omega_{s} V_{s}-\mu_{s} V_{s} \\
\frac{d S_{m}}{d t}=\alpha_{m}+\omega_{m} V_{m}- \\
\frac{d E_{m}}{d t}=\mu_{m} S_{m}-\Psi_{m s} I_{s}-\mu_{m} E_{m}-\rho_{m} E_{m} \\
\frac{d I_{m}}{d t}=\rho_{m} E_{m}-\left(\mu_{m}+\delta_{m}\right) I_{m} \\
\frac{d V_{m}}{d t}=\sigma_{m} S_{m}-\omega_{m} V_{m}-\mu_{m} V_{m}
\end{array}\right.
$$

with,

$$
\begin{gathered}
N_{d}(t)=S_{d}(t)+E_{d}(t)+I_{d}(t)+V_{d}(t) \\
N_{s}(t)=S_{s}(t)+E_{s}(t)+I_{s}(t)+V_{s}(t) \\
N_{m}(t)=S_{m}(t)+E_{m}(t)+I_{m}(t)+V_{m}(t)
\end{gathered}
$$

where $N_{i}, i=d, s, m$ is the total of domestic dogs, stray dogs and Maasai dog's population at time $t$. The Disease Free Equilibrium Point (DFE) was computed and it is given by:

$$
\begin{gathered}
\varepsilon_{0}=\left(\frac{\alpha_{d}-\Psi_{d s}+\Psi_{m d}+\Psi_{s d}}{\mu_{d}+\sigma_{d}}, 0,0,0 \frac{\left(\mu_{s}+\omega_{s}\right)\left(\Psi_{d s}-\Psi_{s d}+\Psi_{m s}+\alpha_{s}\right)}{\mu_{c}\left(\mu_{s}+\omega_{s}\right)+\mu_{s}\left(\mu_{s}+\sigma_{s}+\omega_{s}\right)}, 0,0\right. \\
\left.\frac{\alpha_{s}\left(\Psi_{d s}+\Psi_{m s}+\alpha_{s}-\Psi_{s d}\right)}{\mu_{c}\left(\mu_{s}+\omega_{s}\right)+\mu_{s}\left(\mu_{s}+\sigma_{s}+\omega_{s}\right)}, \frac{\alpha_{m}-\Psi_{m d}-\Psi_{m s}}{\mu_{m}+\sigma_{m}}, 0,0,0\right)
\end{gathered}
$$

\subsection{Sensitivity Analysis}


International Journal of Advances in Scientific Research and Engineering (ijasre),Vol 5 (9), September-2019

Sensitivity analysis helps to determine the most sensitive parameters to the model. This further helps to decide on which parameters to put more effort on combating disease transmission. Now, since we want to understand the dynamics of rabies in Arusha region and therefore control it by targeting the most sensitive parameters, sensitivity analysis will help us by playing a role to determine those parameters. Parameter values used in DFE are as shown below.

Table 1: Values of Parameters Used at DFE

\begin{tabular}{|c|c|c|c|}
\hline Parameter Symbol & Parameter Name & Value & Source \\
\hline$\alpha_{s}$ & The annual births of stray dogs & $2.5 \times 10^{3}$ & [17] \\
\hline$\delta_{s}$ & Death rate due rabies for stray dogs & 0.22 & [19] \\
\hline$\omega_{s}$ & Loss rate of vaccination immunity for stray dogs & 0.1 & Assumption \\
\hline$\mu_{s}$ & Natural death rate of stray dogs & 0.32 & [20] \\
\hline$\beta_{s}$ & Rate of infection of stray dogs & $1.7864 \times 10^{-4}$ & Data \\
\hline$\rho_{s}$ & The incubation period of stray dog & 0.83778234 & [21] \\
\hline$\sigma_{s}$ & Vaccination rate of the susceptible stray dogs & 0.25174 & Data \\
\hline$\Psi_{m s}$ & $\begin{array}{l}\text { Average number of Maasai dogs that migrate to } \\
\text { stray dogs' population }\end{array}$ & 35 & Fitting \\
\hline$\Psi_{s d}$ & $\begin{array}{l}\text { Average number of stray dogs that migrate to } \\
\text { domestic dogs' population }\end{array}$ & 17 & Fitting \\
\hline$\Psi_{d s}$ & $\begin{array}{l}\text { Average number of domestic dogs that migrate to } \\
\text { stray dogs' population }\end{array}$ & 56 & Fitting \\
\hline$\mu_{c}$ & Average culling rate of stray dogs & 0.01792 & Data \\
\hline
\end{tabular}

\subsubsection{Sensitivity Analysis of $R_{e}$}

Sensitivity analysis tells us how important each parameter is to disease transmission and is used to assess how sensitive a model is to variation in the value of the parameters of the model and to changes in the structure of the model [4]. In this case, the normalized forward sensitivity index was employed by using the MATHEMATICA program. The normalized forward sensitivity index is the ratio of relative change of a variable to the relative change in parameter. If the variable is a differentiable function of the parameter then the sensitivity index is defined as follows:

Definition 1: The normalized forward sensitivity index of variable $\mathrm{V}$ that depends on parameter $\rho$ is defined as;

$$
S_{\rho}^{V}=\frac{\partial V}{\partial \rho} \times \frac{\rho}{V}
$$

For example in our case, we have the effective reproduction number $R_{e}$ computed. The normalized forward sensitivity with respect to the parameter $\rho$ is given by:

$$
S_{\rho}^{R_{e}}=\frac{\partial R_{e}}{\partial \rho} \times \frac{\rho}{R_{e}}
$$

For instance, the sensitivity index of $R_{e}$ with respect to parameter $\beta_{s}$ is given by:

$$
S_{\beta_{s}}^{R_{e}}=\frac{\partial R_{e}}{\partial \beta_{s}} \times \frac{\beta_{S}}{R_{e}}=+1
$$

By using the same idea, the sensitivity indices of $R_{e}$ given by the expression below;

$$
R_{e}=\frac{\beta_{s} \rho_{s}\left(\mu_{s}+\omega_{s}\right)\left(\Psi_{d s}+\Psi_{m s}+\alpha_{s}-\Psi_{s d}\right)}{\left(\mu_{s}+\delta_{s}\right)\left(\mu_{s}+\rho_{s}\right)\left(\mu_{c} \omega_{s}+\mu_{s} \mu_{c}+\mu_{s} \sigma_{s}+\mu_{s} \omega_{s}+\mu_{s}^{2}\right)}
$$

is computed with respect to all parameters fixed to $R_{e}$ and are as shown in the table below. 
International Journal of Advances in Scientific Research and Engineering (ijasre),Vol 5 (9), September-2019

Table 2: Sensitivity Indices of $\boldsymbol{R}_{\boldsymbol{e}}$

\begin{tabular}{|c|c|}
\hline Parameter & Sensitivity Index Value \\
\hline$\alpha_{s}$ & +0.9713 \\
\hline$\delta_{s}$ & -0.4074 \\
\hline$\omega_{s}$ & +0.0866 \\
\hline$\mu_{s}$ & -1.5593 \\
\hline$\beta_{s}$ & +1 \\
\hline$\rho_{s}$ & +0.2764 \\
\hline$\sigma_{s}$ & -0.3621 \\
\hline$\Psi_{m s}$ & +0.0136 \\
\hline$\Psi_{s d}$ & $-6.6045 \times 10^{-3}$ \\
\hline$\Psi_{d s}$ & +0.0218 \\
\hline$\mu_{c}$ & -0.0338 \\
\hline
\end{tabular}

According to the sensitivity indices, infection rate of stray $\operatorname{dogs} \beta_{s}$ is the most positive sensitive parameter followed by the annual births of stray $\operatorname{dogs} \alpha_{s}$ and the incubation period of stray $\operatorname{dogs} \rho_{\mathrm{s}}$. This means, increasing these parameters, will result to increase in the effective reproduction number $\mathrm{R}_{\mathrm{e}}$. For example, increasing $\beta_{\mathrm{s}}$ by $10 \%$ will result to increase in $\mathrm{R}_{\mathrm{e}}$ by $10 \%$ also decreasing $\beta_{\mathrm{s}}$ by $10 \%$ will result to decrease in $\mathrm{R}_{\mathrm{e}}$ by $10 \%$. Average number of Maasai dogs that migrate to stray dogs' population $\Psi_{\mathrm{ms}}$, loss rate of vaccination immunity of stray $\operatorname{dogs} \omega_{\mathrm{s}}$ and average number of domestic dogs that migrate to stray dogs' population $\Psi_{\mathrm{ds}}$ are the less positively sensitive parameters.

Also, natural death rate of stray dogs $\mu_{\mathrm{s}}$ is the most negative sensitive parameter followed by death rate for stray dogs due to rabies $\delta_{\mathrm{s}}$ and vaccination rate of the susceptible stray $\operatorname{dogs} \sigma_{\mathrm{s}}$. These imply that increase in these parameters will result to decrease in the effective reproduction number $R_{e}$. For instance, increase in natural death rate $\mu_{s}$ by $10 \%$ results to decrease in Re by approximately $16 \%$. Average number of stray dogs that migrate to domestic dogs' population $\Psi_{\text {sd }}$ and average culling rate of stray $\operatorname{dogs} \mu_{\mathrm{c}}$ are the less negatively sensitive parameters.

We can deduce that, putting much emphasis on the most positive and most negative sensitive parameter will be the most effective way in combating dog-rabies transmission in Arusha region.

\subsection{Numerical Simulations Results and Discussion}

In this section, several results and the interpretation have been presented. We used ode45 MATLAB's standard solver for ordinary differential equations (ODEs). In this function a Runge-Kutta method with a variable time step for efficient computation is implemented. 2013 to 2018 reported data from the ministry of Agriculture, Livestock Development and Fisheries of the United Republic of Tanzania and also survey data from Mbwa wa Africa were used. Information on the number of dogs that migrate from one population to another was missing so the parameters were obtained through data fitting. 
International Journal of Advances in Scientific Research and Engineering (ijasre),Vol 5 (9), September-2019

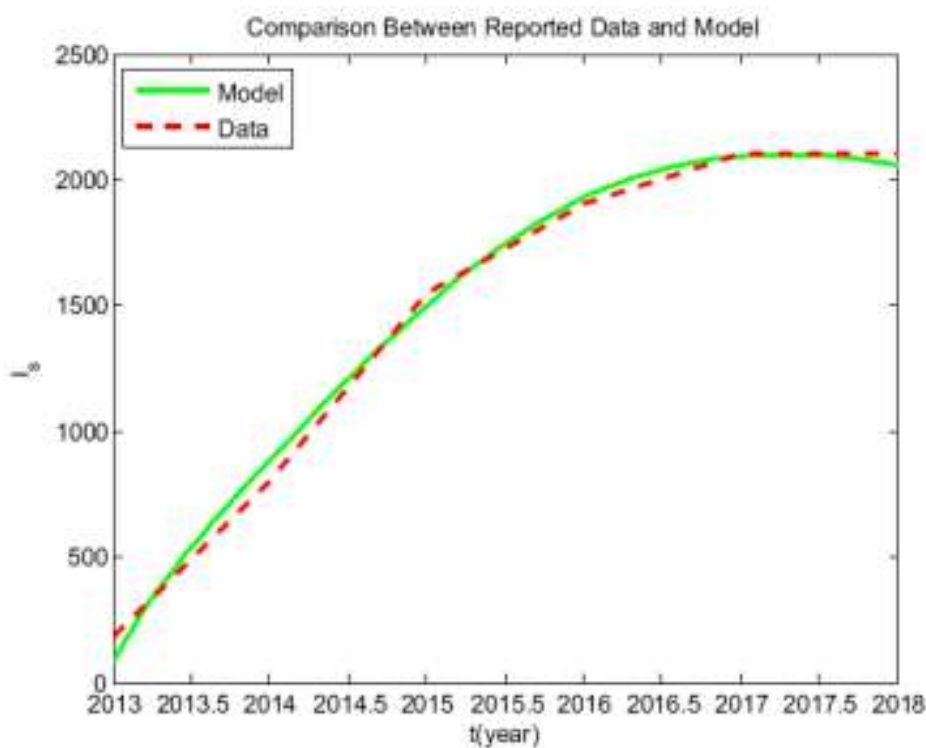

Figure 1: Details of manuscript font size Comparison between reported data and simulation of system 1 for rabies infected stray dogs in Arusha region from 2013 to 2018

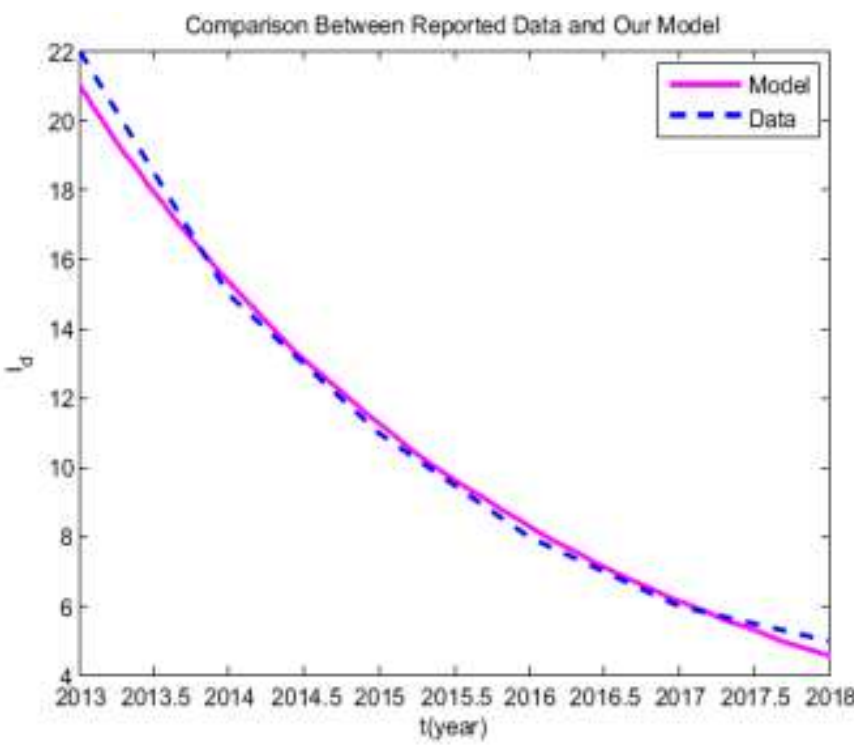

Figure 2: Comparison between reported data and simulation of system 1 for rabies infected domestic dogs in Arusha region from 2013 to 2018

From the figure 1 above, we have tried to fit our data for infectious stray dogs into the model; from the year 2013 to 2018 . We compare the reported data and the simulation of our model in the system of differential equations. The dashed red line is for our data and the full green line is the simulation of our model system. We see that there is a good match between the reported data and our model. Also, our model predicts that, the number of infectious stray dogs will increase but later on will decrease because the infected stray dogs due to rabies will later on die. The initial conditions of the variables are consisting of the data that are reported from the ministry of Agriculture, Livestock Development and Fisheries of the United Republic of Tanzania and Mbwa wa Africa, logical assumptions and data fitting.

Using the same initial conditions, we fitted the data for rabies infected domestic dogs from the year 2013 to 2018 into the model. Also from the figure 2 above we see that the number of rabies infected domestic dogs will be decreasing. This is because the infected dogs will die since rabies is incurable. The full magenta line indicates the model and the dotted blue line stands for our data. We also observe that there is a good match between our model the reported data.

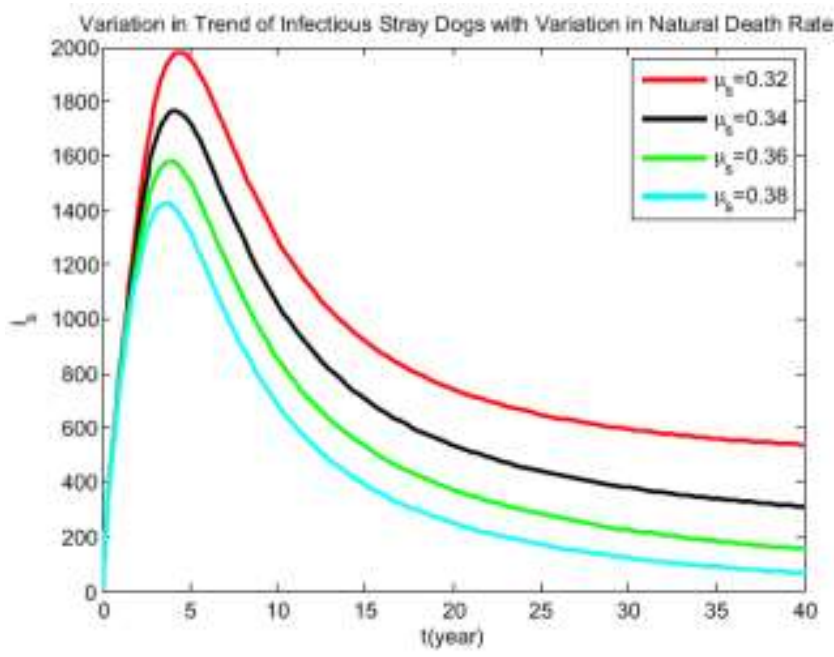

Figure 3: The effect of natural death rate of stray dogs to stray dogs rabies infection

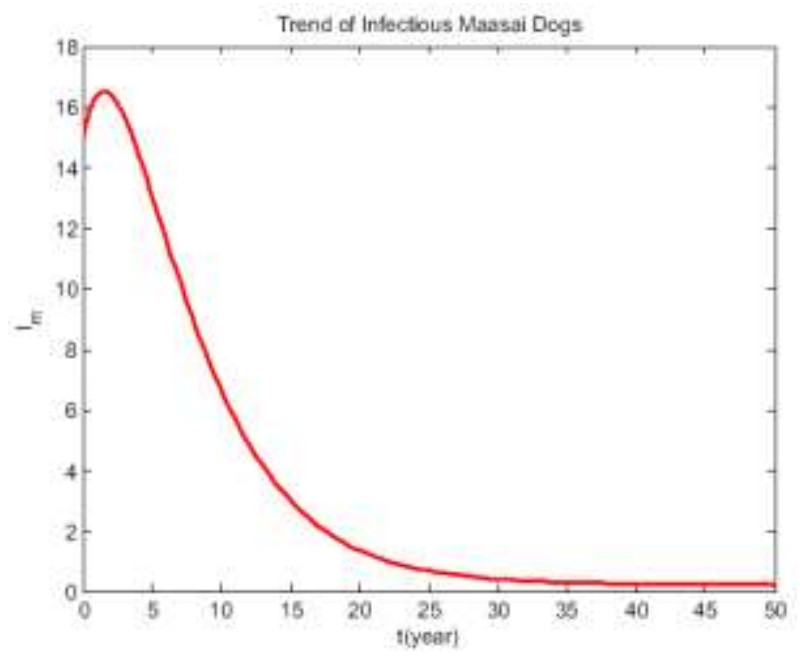

Figure 4: Trend of infected Maasai dogs

From the sensitivity analysis we found that, natural death rate is the most sensitive parameter in controlling the dynamics of dog rabies transmission. Figure 3 above shows how a minor increase in the natural death rate of stray dogs results to decrease in the number of infectious stray dogs and vice versa. It can be noted that, controlling deaths due to rabies through vaccination will encourage natural deaths of stray dogs. This again insists vaccination of stray dogs as one of the most effective way to reduce dog rabies transmission in Arusha region. 
From figure 4 above, we see that the population of infectious Maasai dogs will increase rapidly and it will peak in 2022 . The increase is because exposed Maasai dogs will move to infectious group once they develop symptoms of rabies and hence it will result to increase in the infectious group. Later on, this group will decline in number since they will die as observed in a period of 50 years.

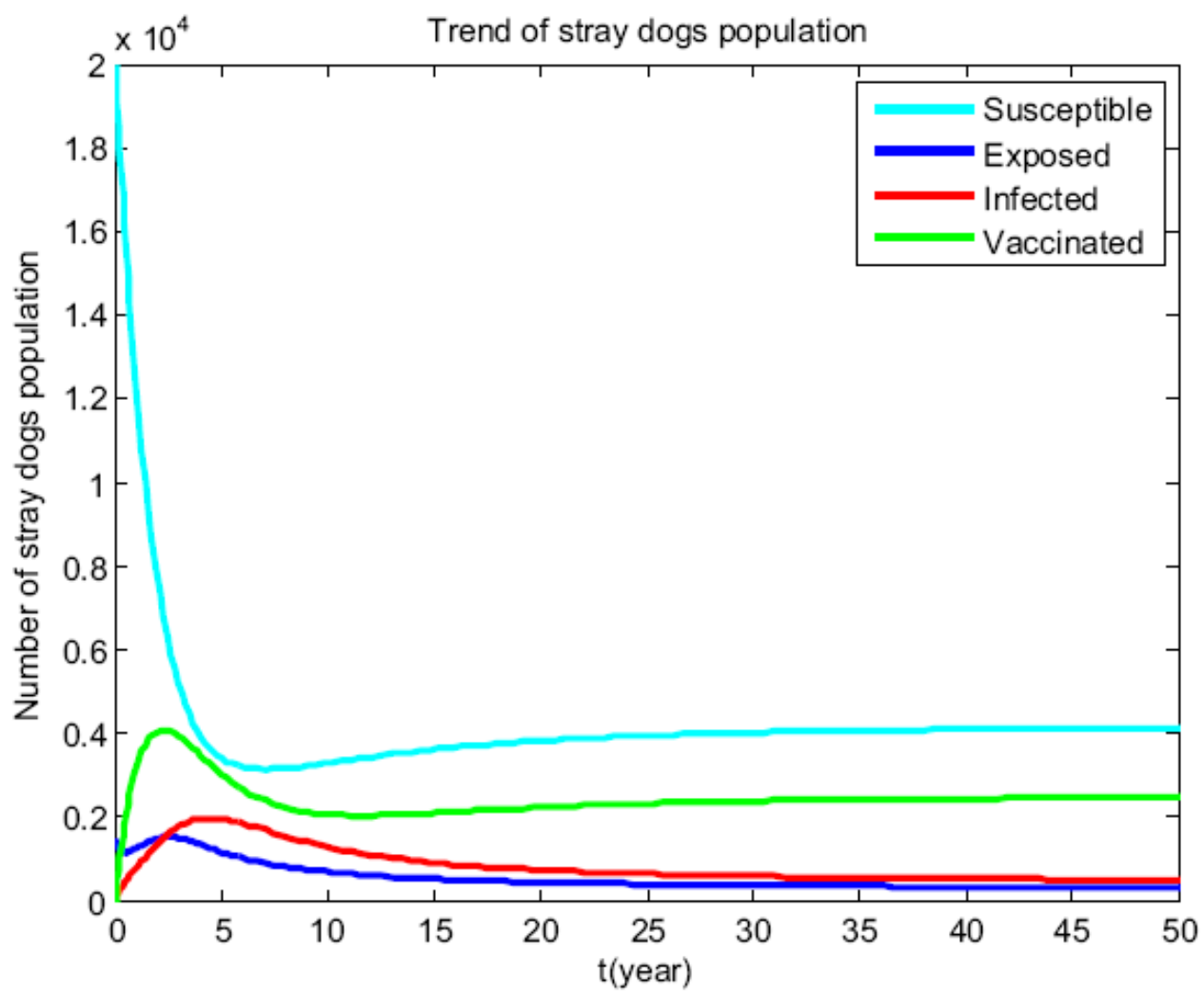

Figure 5: Trend stray dogs' population for a period of 50 years

From the figure 5 above we see that the group of susceptible stray dogs will decline because once a susceptible dog is attacked and scratched or bitten, it become exposed. This result to increase in the number of exposed stray dogs. Before exposed stray dogs develop symptoms, if they are vaccinated they shift to vaccination group but if not, they become infectious and later on they die. So due to this we can see that the exposed group will later on decline and so the infectious group will increase in number and after some time the group will decline because rabies is incurable so the infected dogs will die. Also the vaccinated group will decline because when the exposed stray dogs are vaccinated and shift to vaccination group, the vaccinated stray dogs become susceptible.

\section{CONCLUSION}

The current strategy of culling stray dogs which is administered by Arusha region is not ideal in controlling the transmission of rabies as compared to the strategy proposed in this study.

Based on the sensitivity analysis results, putting much emphasis on the most positive and most negative sensitive parameter will be the most effective way in combating dog-rabies transmission in Arusha region.

The numerical simulations of the basic model formulated in this study show that, in 2022 there will be more than 17 cases of rabies infected Maasai dogs. Also, number of rabies infected domestic dogs will decline to its minimum in 2020 because there is an increase in number of deaths due to rabies for domestic dogs. Further, number of infected stray dogs will peak in 2023 whereby there will be more than 2000 rabies infected stray dogs. The increased number of rabies infected stray and Maasai dogs will later on decline because the infected dogs will die.

\section{ACKNOWLEDGMENT}

The authors thank MWECAU for the sponsorship of studies. Also authors appreciate the data provision support from the ministry of agriculture, livestock development and fisheries of the United Republic of Tanzania and Dr. Jens Fissernbert of Mbwa wa Africa. 
International Journal of Advances in Scientific Research and Engineering (ijasre),Vol 5 (9), September-2019

\section{CONFLICT OF INTEREST}

Authors declare that there is no conflict of interest

\section{REFERENCES}

1. R. S. Mani, S. N. Madhusudana, "Laboratory diagnosis of human rabies: recent advances" The Scientific World Journal 2013 (2013).

2. G. Gongal, A. E. Wright, "Human rabies in the WHO Southeast Asia region: forward steps for elimination" Advances in preventive medicine 2011 (2011).

3. W. H. Wunner, A. C. Jackson, "Rabies: scientific basis of the disease and its management" Academic Press, 2010.

4. O. Peter, A. Ayoade, A. Abioye, A. Victor, C. Akpan, "Sensitivity analysis of the parameters of a cholera model", Journal of Applied Sciences and Environmental Management 22 (2018) 477-481.

5. K. Hampson, J. Dushoff, S. Cleaveland, D. T. Haydon, M. Kaare, C. Packer, A. Dobson, "Transmission dynamics and prospects for the elimination of canine rabies"PLoS biology 7 (2009) e1000053.

6. T. Lembo, K. Hampson, M. T. Kaare, E. Ernest, D. Knobel, R. R. Kazwala, D. T. Haydon, S. Cleaveland, "The feasibility of canine rabies elimination in africa: dispelling doubts with data"PLoS neglected tropical diseases 4 (2010) e626.

7. J. Zhang, Z. Jin, G.-Q. Sun, T. Zhou, S. Ruan, “Analysis of rabies in China: transmission dynamics and control”PLoS one 6 (2011) e20891.

8. Q. Hou, Z. Jin, S. Ruan, "Dynamics of rabies epidemics and the impact of control efforts in guangdong province, China" Journal of theoretical biology 300 (2012) 39-47.

9. J. P. Keller, L. Gerardo-Giorda, A. Veneziani, "Numerical simulation of a susceptible-exposed-infectious spacecontinuous model for the spread of rabies in raccoons across a realistic landscape" Journal of biological dynamics 7 (2013) 31-46.

10. T. T. Ega, L. S. Luboobi, D. Kuznetsov, "Modeling the dynamics of rabies transmission with vaccination and stability analysis" Applied and Computational Mathematics 4 (2015) 409-419.

11. A. Abta, H. Laarabi, H. TalibiAlaoui, "The hopf bifurcation analysis and optimal control of a delayed sir epidemic model" International Journal of Analysis 2014 (2014).

12. S. A. Elmore, R. B. Chipman, D. Slate, K. P. Huyvaert, K. C. VerCauteren, A. T. Gilbert, "Management and modeling approaches for controlling raccoon rabies: The road to elimination"PLoS neglected tropical diseases 11 (2017) $\mathrm{e} 0005249$.

13. S. Ruan, "Spatiotemporal epidemic models for rabies among animals" Infectious Disease Modelling 2 (2017) $277-287$.

14. M. Laager, C. Mbilo, E. A. Madaye, A. Naminou, M. L’echenne, A. Tschopp, T. Smieszek, J. Zinsstag, N. Chitnis,et al., "The importance of dog population contact network structures in rabies transmission"PLoS neglectedtropical diseases 12 (2018) e0006680.

15. N. N. Chidumayo, "System dynamics modelling approach to explore the effect of dog demography on rabiesvaccination coverage in Africa"PloS one 13 (2018) e0205884.

16. S. D“urr, N. Dhand, C. Bombara, S. Molloy, M. Ward, "What influences the home range size of free-roaming domestic dogs?" Epidemiology \& Infection 145 (2017) 1339-1350.

17. S. C. Totton, A. I.Wandeler, J. Zinsstag, C. T. Bauch, C. S. Ribble, R. C. Rosatte, S. A. McEwen, "Stray dog population demographics in jodhpur India following a population control/rabies vaccination program" Preventive veterinary medicine 97 (2010) 51-57.

18. S. Cleaveland, T. Mlengeya, M. Kaare, D. Haydon, T. Lembo, M. K. Laurenson, C. Packer, "The conservation relevance of epidemiological research into carnivore viral diseases in the Serengeti” Conservation Biology 21 (2007) 612-622.

19. M. Amaku, R. A. Dias, F. Ferreira, "Dynamics and control of stray dog populations, Mathematical Population Studies" 17 (2010) 69-78.

20. M. Paul, S. S. Majumder, S. Sau, A. K. Nandi, A. Bhadra, "High early life mortality in free-ranging dogs is largely influenced by humans" Scientific reports 6 (2016) 19641.

21. T. Leung, S. A. Davis, "Rabies vaccination targets for stray dog populations" Frontiers in veterinary science 4 (2017) 52. 